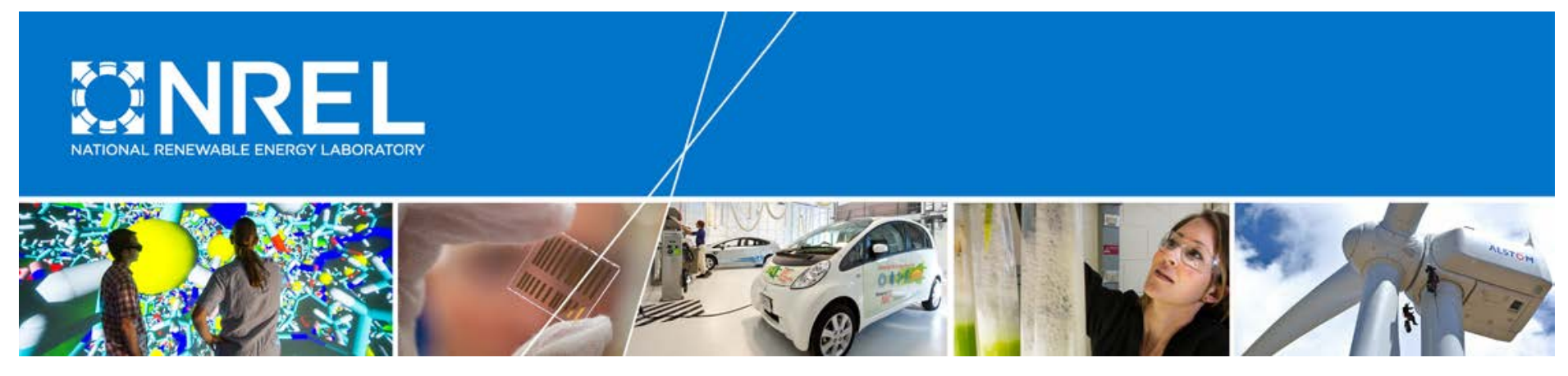

\title{
Efficient Optimization of Large Wind Farms for Real-Time Control
}

\section{Preprint}

Jennifer Annoni, Paul Fleming, and Kathryn Johnson

National Renewable Energy Laboratory

Christopher Bay and Timothy Taylor Colorado School of Mines

\section{Lucy Pao}

University of Colorado - Boulder

Presented at American Control Conference

Milwaukee, Wisconsin

June 27-29, 2018

\section{Suggested Citation}

Annoni, Jennifer, Paul Fleming, Kathryn Johnson, Christopher Bay, Timothy Taylor, and Lucy Pao. 2018. "Efficient Optimization of Large Wind Farms for RealTime Control: Preprint." Golden, CO: National Renewable Energy Laboratory. NREL/CP-5000-70937. https://www.nrel.gov/docs/fy18osti/70937.pdf.

NREL is a national laboratory of the U.S. Department of Energy Office of Energy Efficiency \& Renewable Energy Operated by the Alliance for Sustainable Energy, LLC

This report is available at no cost from the National Renewable Energy Laboratory (NREL) at www.nrel.gov/publications.

\section{Conference Paper}

NREL/CP-5000-70937

September 2018 


\section{NOTICE}

This work was authored in part by the National Renewable Energy Laboratory, operated by Alliance for Sustainable Energy, LLC, for the U.S. Department of Energy (DOE) under Contract No. DE-AC36-08G028308. Funding provided by U.S. Department of Energy Office of Energy Efficiency and Renewable Energy Wind Energy Technologies Office. The views expressed in the article do not necessarily represent the views of the DOE or the U.S. Government. The U.S. Government retains and the publisher, by accepting the article for publication, acknowledges that the U.S. Government retains a nonexclusive, paid-up, irrevocable, worldwide license to publish or reproduce the published form of this work, or allow others to do so, for U.S. Government purposes.

This report is available at no cost from the National Renewable Energy Laboratory (NREL) at www.nrel.gov/publications.

U.S. Department of Energy (DOE) reports produced after 1991 and a growing number of pre-1991 documents are available free via www.OSTI.gov. 


\title{
Efficient Optimization of Large Wind Farms for Real-Time Control
}

\author{
Jennifer Annoni, ${ }^{1}$ Christopher Bay, ${ }^{2,3}$ Timothy Taylor,${ }^{2}$ Lucy Pao, ${ }^{3}$ Paul Fleming, ${ }^{1}$ Kathryn Johnson ${ }^{1,2}$
}

\begin{abstract}
Wind turbines in a wind farm typically operate individually to maximize their own performance regardless of the impact of aerodynamic interactions on neighboring turbines. Properly coordinating turbines, by operating some turbines suboptimally, within a wind farm has the potential to improve overall wind farm performance. Computing the optimal control strategy under varying atmospheric conditions can be computationally intense for large wind farms. As wind power farms increase in size and related models become more complex, computationally efficient algorithms are needed to perform real-time optimization and control. This study proposes a distributed optimization framework and computationally efficient wake steering wind farm control strategy that uses the yaw angle of a turbine to alter the behavior of a turbine wake and minimize turbine interactions. This computational efficiency allows the strategy to be feasible for real-time control.
\end{abstract}

\section{INTRODUCTION}

Wind farm control can be used to achieve a number of objectives including increasing power production in a wind farm and the lifetime of turbines in a wind farm, and tracking power reference signals to improve wind integration into the energy grid. This paper focuses on increasing the power production of a wind farm by operating some wind turbines suboptimally to improve the performance of the entire wind farm [1]-[4]. Many studies have shown that operating all turbines at their own locally optimal operating point leads to suboptimal global performance [5], [6]. One common wind power plant control strategy in literature is known as wake redirection or wake steering. There has been a significant

*This work was supported by the U.S. Department of Energy under Contract No. DE-AC36-08GO28308 with the National Renewable Energy Laboratory. Funding for the work was provided by the DOE Office of Energy Efficiency and Renewable Energy, Wind Energy Technologies Office. The U.S. Government retains and the publisher, by accepting the article for publication, acknowledges that the U.S. Government retains a nonexclusive, paid-up, irrevocable, worldwide license to publish or reproduce the published form of this work, or allow others to do so, for U.S. Government purposes. The authors are solely responsible for any omission or errors contained herein. In addition, this work has been partially supported by ARPA-E under award number DE-AR0000667, the German Academic Exchange Service (DAAD) with funds from the Federal Ministry of Education and Research (BMBF), and the National Science Foundation under Grant No. NSF-CMMI-1234980. Any opinions, findings, and conclusions or recommendations expressed in this material are those of the author(s) and do not necessarily reflect the views of the funding agencies. Lastly, this material is based on work supported by Envision Energy. Any opinions, findings, and conclusions or recommendations expressed in this material are those of the author(s) and do not necessarily reflect the views of Envision Energy.

$\mathrm{J}$. Annoni is a research engineer at the National Renewable Energy Laboratory (e-mail: jennifer.annoni@nrel.gov).

${ }^{1}$ National Renewable Energy Laboratory, Golden, CO 80401 USA. ${ }^{2}$ Electrical Engineering Department, Colorado School of Mines, Golden, CO, 80401 USA. ${ }^{3}$ Electrical, Computer, \& Energy Engineering, University of Colorado, Boulder, CO, 80309, USA. amount of work done on wake redirection that shows this method has potential to increase power production [2], [3], [7], [8]. Wake redirection typically uses the yaw drive of the turbines to redirect the flow around downstream turbines. Various computational fluid dynamics simulations and wind tunnel experiments have shown that this method can increase power without substantially increasing turbine loads [3], [9], [10]. Standard wind farm control approaches use a look-up table based on offline optimization results [7]. This approach breaks down when individual turbines are unavailable due to maintenance. For small wind farms, an optimization can be performed in real time and adapt to changing atmospheric and turbine conditions. However, as wind farms increase in size, computationally efficient algorithms are needed to perform real-time optimization and control.

Distributed optimization and control provides a framework for efficient computation of large systems. For example, these types of controls have been used for multiagent systems and can be used to coordinate subsystems to interact with their larger environment [11]-[13]. This approach has also been used to evaluate the optimal power flow on the grid [14] Distributed optimization has also been considered in wind farm controls literature [15]-[17]. However, the complex aerodynamic interactions and large timescales make this a challenging problem. For example, a distributed optimization framework for wind farm controls has been presented by [18]. Yet, solving this problem becomes computationally complex as the system grows because of the number of turbines and larger flow domains. A limited-communication distributed model predictive controller is described in [19] to track a power reference signal, which uses a simplified linearized wake model to describe turbine interactions, allowing for scalability.

This paper focuses on a distributed optimization framework that enables the real-time optimization that is much more difficult to achieve using centralized optimization techniques. In this paper, the wind farm is modeled using a nonlinear steady-state model described in Section II-A. Typically, the optimization problem is formulated as a global optimization, including all of the turbines in a wind farm, as described in Section II-B. However, this paper demonstrates that a wind farm can be modeled as a distributed system by dividing it into subsets based on wake interactions. This distributed problem is solved using the alternating direction method of multipliers (ADMM) [20], described in Section III. Next, this distributed optimization framework is demonstrated via simulation of the Princess Amalia offshore wind farm, consisting of 60 turbines (Section IV) and presented in previous studies [21]. The results show a 
significant reduction in computation time without sacrificing the overall power gain of the wind farm when comparing finite-difference gradient-based techniques, shown in Section V. Finally, we conclude by discussing the implications of increased computational efficiency and propose future work in Section VI.

\section{BACKGROUND}

This section briefly describes the wind turbine wake model used in this paper as well as the full optimization problem used for wake redirection control.

\section{A. Wind Turbine Wake Model}

A wind turbine wake model is used to characterize the aerodynamic interactions in a wind farm. The model used for this work was introduced by several recent papers including [22]-[26]. This model uses a Gaussian wake shape to describe the velocity deficit behind a turbine and includes the effects of turbulence in the wake and local atmospheric conditions. The Gaussian wake is based on self-similarity theory used in free shear flows [27]. An analytical expression for the three-dimensional velocity deficit behind the turbine in the far wake can be derived from the simplified NavierStokes equations:

$$
\frac{u(x, y, z)}{U_{\infty}}=1-C e^{-(y-\delta)^{2} / 2 \sigma_{y}^{2}} e^{-\left(z-z_{h}\right)^{2} / 2 \sigma_{z}^{2}}
$$

where $u$ is the velocity in the wake, $U_{\infty}$ is the free-stream velocity, $x$ is the streamwise direction, $y$ is the spanwise direction, $\delta$ is the wake centerline, $z$ is the vertical direction, $z_{h}$ is the hub height, $\sigma_{y}$ is the wake expansion in the $y$ direction, $\sigma_{z}$ is the wake expansion in the $z$ direction, and $C$ is the velocity deficit at the wake center. These parameters are further defined in [23].

A wake deflection model used to describe the turbine behavior in misaligned conditions is also implemented based on [23]. The wake deflection angle at the rotor is defined as:

$$
\alpha \approx \frac{0.3 \gamma}{\cos \gamma}\left(1-\sqrt{1-C_{T} \cos \gamma}\right)
$$

where $\gamma$ is the yaw angle of the turbine and $C_{T}$ is the thrust coefficient determined by turbine operating parameters, such as blade pitch and generator torque. The initial wake deflection, $\delta_{0}$, is then defined as:

$$
\delta_{0}=x_{0} \tan \alpha
$$

where $x_{0}$ indicates the length of the near wake, which is typically on the order of 3 rotor diameters. A full description of the wake deflection can be found in [23].

Lastly, a turbine model is used in this wake model to provide a realistic description of turbine interactions in a wind farm. The turbine model consists of a power coefficient, $C_{P}$, and thrust coefficient, $C_{T}$, based on wind speed and constant blade pitch angle. The coupling between $C_{P}$ and $C_{T}$ is critical in understanding the benefits of wind farm controls. In other words, each turbine is free to operate at its own $C_{P}$ and $C_{T}$ based on local conditions.

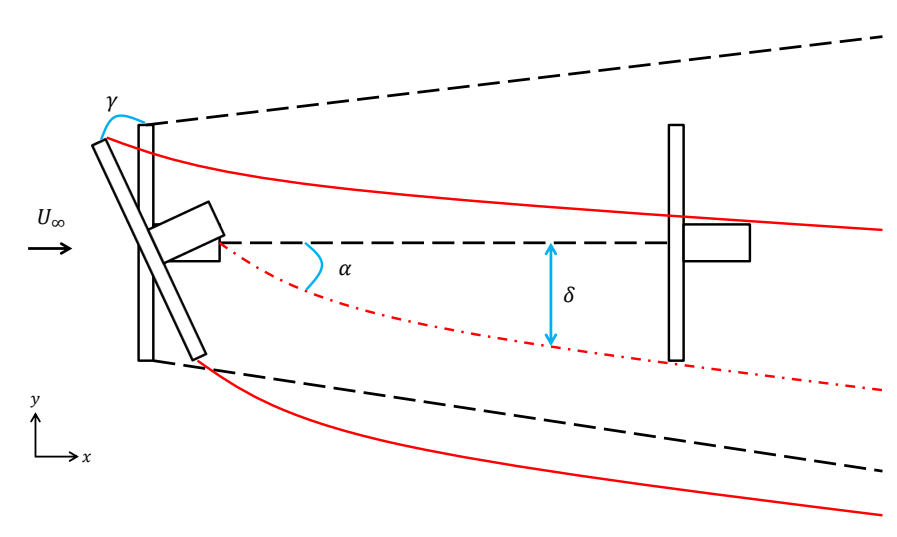

Fig. 1: Two-turbine example of wake redirection control, where $\gamma$ denotes the yaw angle of the upstream turbine, $\alpha$ denotes the deflection angle, and $\delta$ denotes the wake deflection. The black dashed lines represent the wake of the upstream turbine under nonyawed conditions and the red lines denote the wake of the upstream turbine under yawed conditions.

The steady-state power of each turbine under yaw misalignment conditions is given by [28]:

$$
P=\frac{1}{2} \rho A C_{P}(\cos \gamma)^{p} u^{3}
$$

where $\rho$ is the air density, $A$ is the rotor area, $\cos \gamma^{p}$ is a correction factor added to account for the effects of yaw misalignment, and $p$ is a tuneable parameter that matches the power loss caused by the yaw misalignment seen in simulations. In actuator disk theory [28], $p=3$. However, based on large-eddy simulations, the turbine power in yaw misalignment has been shown to match the output with $p=1.88$ when using the National Renewable Energy Laboratory's 5-MW wind turbine [2].

\section{B. Optimal Wind Farm Control}

As stated previously, wake steering or wake redirection control uses the yaw drive of a turbine to deflect a turbine's wake away from the downstream turbine. This section describes the centralized yaw optimization problem for a twoturbine array, shown in Fig. 1. In practice, this is extended to many turbines in a wind farm.

$P_{1}$ and $P_{2}$ denote the power from the upstream turbine and downstream turbine, respectively. In this study, the blade pitch and generator torque of the turbines operate at the optimal operating point of the individual turbine [29]. The power generated by the upstream turbine depends on the local inflow wind speed, $U_{\infty}$, and its yaw angle, $\gamma_{1}$. The power generated can be expressed using Eq. (4). Therefore, the power generated by the upstream turbine can be expressed as a function of the inflow velocity and the yaw angle, $P_{1}\left(\gamma_{1}, U_{\infty}\right)$. Because the yaw angle of the upstream turbine can be used to steer the wake into or away from the downstream turbine, the power of the second turbine is now a function of the yaw angle of the upstream turbine, $\gamma_{1}$. The power generated by the downstream turbine 
is now expressed as $P_{2}\left(\gamma_{1}, \gamma_{2}, u\right)$, where $u$ is the disturbed local incoming velocity to the downstream turbine. The total power generated by the two-turbine array is given by:

$$
P_{\text {tot }}\left(\gamma, U_{\infty}, u\right)=P_{1}\left(\gamma_{1}, U_{\infty}\right)+P_{2}(\gamma, u)
$$

where the vector $\gamma:=\left[\begin{array}{ll}\gamma_{1} & \gamma_{2}\end{array}\right]^{T}$. A similar approach can be applied for an $N$-turbine array, where the power of each turbine can be written as $P_{i}\left(\gamma, U_{\infty}, u\right)$, where $\gamma$ consists of yaw angles of all upstream turbines. The main objective of wake redirection is to maximize the total average power output:

$$
\underset{\gamma}{\operatorname{minimize}} f(\gamma)=-P_{t o t}(\gamma, u)
$$

where $\gamma$ includes all $N$ turbines. This optimization problem assumes a constant, free-stream velocity, $U_{\infty}$, which is a steady-state formulation. Future work will include a more realistic inflow velocity in which the free-stream velocity is unsteady and turbulent. In this paper, the objective is to maximize the steady-state power generated by the wind farm. However, yaw misalignment can cause significant structural loads to the turbines, thereby the optimization problem presented in this section can be extended to include constraints on the loads. The inclusion of loads is considered outside the scope of this paper.

It should be noted that, for small wind farms, this optimization can be computed in real time and adapt to changing atmospheric conditions. However, as wind farms increase in size, computationally efficient algorithms are needed to perform real-time optimization and control.

\section{Distributed OPTIMIZATION OF Wind FARMS}

In this section, we describe a distributed optimization framework that can significantly reduce computation time by taking advantage of the spatial distribution of the turbines within a wind farm. This section describes the procedure for setting up and solving this distributed optimization problem.

\section{A. Wind Farm Network}

A wind energy farm can be represented as a directed network, wherein each turbine represents a node in the network and the aerodynamic interactions between turbines can be represented as edges of this directed network. The wind farm is a directed network because actions of the upstream turbines affect the downstream turbines, but downstream turbine actions do not affect upstream turbines. A weight is typically assigned to each edge indicating the strength of interactions between nodes [30]. For the wind farm problem, the weight of each edge is assigned based on the strength of the wake impacting the downstream turbine. The weights are determined by three factors: (1) distance downstream, (2) area overlap based on thresholding, and (3) operating points of the upstream turbines. The operating point of an upstream turbine determines the wake characteristics and can significantly affect the strength and size of the wake. The edge weights are defined as:

$$
w_{i, j}=\frac{A_{\text {overlap }}}{x / D}
$$

where $i$ denotes the upstream turbine, $j$ denotes the downstream turbine, $A_{\text {overlap }}$ represents the overlap of the wake of turbine $i$ to the rotor disk of turbine $j, x$ is the downstream distance between turbines $i$ and $j$, and $D$ is the rotor diameter assumed to be the same for all turbines. Depending on turbine operation, the wake can expand faster or slower, which affects the area overlap of the wake at the downstream turbine. Future work will consider the strength of the wake as a part of the weighting definition.

Based on these weights, a wind farm can be divided into subsets. Subsets can be determined by many different approaches including nearest neighbor, connectivity, hierarchical, or k-means algorithms [31]. To define the members of each subset, the magnitude of the interactions between turbines, $w_{i j}$, is considered. Specifically, a lead turbine of a subset is identified as a turbine experiencing free-stream velocity, $U_{\infty}$. The turbines that are impacted by the lead turbine are combined into a subset based on their weights. A turbine can belong to multiple subsets depending on the strength of the interaction between wind turbines. By defining smaller subsets, large optimization problems can be solved in a distributed, tractable way.

\section{B. Distributed Optimal Wind Farm Control Problem}

Once the turbines are grouped into subsets, a distributed optimization algorithm is used to solve the optimization problem. In this study, the power throughout the wind farm is maximized among subsets:

$$
\underset{\gamma}{\operatorname{minimize}} \sum_{n}^{N_{s}} f\left(\gamma_{n}\right)=-P_{n}(\gamma, u)
$$

where $n$ refers to different subsets in the wind farm, $N_{s}$ represents the total number of subsets identified in the wind farm, and $\gamma_{n}$ denotes the yaw angles of all of the turbines in the $n$-th subset. Each subset of turbines has a fraction of the number of turbines in the wind farm and each subset can solve their own optimization problem independently in significantly less time than it takes to run a full optimization of the large wind farm. In addition, each subset optimization can be solved in parallel, further reducing computational cost. If the subsets share a node, as is likely in the wind farm example, then an iterative approach that takes into account these shared nodes is implemented. This approach is detailed in the next section.

\section{Alternating Direction Method of Multipliers Algorithm}

ADMM is a popular technique used in literature to solve distributed optimization problems [20]. This algorithm is particularly useful in this case, as each individual subset solves its own optimization, communicates the solution to neighboring subsets, and iterates this process until the wind farm has converged and each shared node has reached a single value. For example, if two subsets contain the same turbine, this algorithm converges to one yaw angle for that turbine. This algorithm was adopted from [32], where ADMM is used to solve a network optimization with 
connecting nodes to determine a consensus between the shared nodes.

Equation (8) does not account for shared nodes between subsets. This section extends Eq. (8) to include a penalty term on the shared nodes such that the shared nodes eventually agree on a value and the network as a whole converges. Thus, the distributed wind farm optimization problem is posed as:

$\operatorname{minimize} \sum_{n}^{N_{s}}\left(f_{n}\left(\gamma_{n}\right)+\lambda \sum_{p}^{N_{s}} \sum_{k \in \Omega_{n p}}\left\|\gamma_{k}-z_{k}\right\|_{2}\right)$

subject to $\gamma_{k}=z_{k}, \quad$ where $\quad \Omega_{n p}=\left\{T_{n} \mid T_{n} \in \Omega_{n} \cap \Omega_{p}\right\}$

where $f\left(\gamma_{n}\right)$ is the power of each cluster, $N_{s}$ is the number of subsets, $\gamma_{n}$ are the yaw angles of all the turbines each subset $n, \lambda$ is the user-defined constant that penalizes shared nodes, $p$ defines a subset that shares a node with subset $n$, and $k$ denotes the individual turbine that is shared between subset $n$ and $p . \Omega_{n j}$ describes the nodes that are shared between each subset and $T_{i}$ represents the turbine number in the wind farm. Lastly, $z$ is a copy of $\gamma$, such that we can ensure that the duplicate nodes converge to the same value.

The distributed optimization problem in Eq. (9) is solved iteratively using ADMM by minimizing the augmented Lagrangian:

$$
\begin{aligned}
\mathcal{L}_{\rho}(\gamma, z, u) & =\sum_{n}^{N_{s}}\left(f_{n}\left(\boldsymbol{\gamma}_{n}\right)+\lambda \sum_{p}^{N_{s}} \sum_{k \in \Omega_{n j}}\left\|\gamma_{k}-z_{k}\right\|_{2}\right) \\
& +\sum_{n}^{N_{s}}\left((\rho / 2)\left\|\boldsymbol{\psi}_{n}\right\|_{2}^{2}+(\rho / 2)\left\|\boldsymbol{\gamma}_{n}-\boldsymbol{z}_{n}+\boldsymbol{\psi}_{n}\right\|_{2}^{2}\right)
\end{aligned}
$$

separately with respect to $\gamma$ and $z$ followed by an update of the scaled dual variable, $\psi$ :

$$
\begin{aligned}
& \gamma^{m+1}=\underset{\gamma}{\operatorname{argmin}} \quad \mathcal{L}_{\rho}\left(\gamma, z^{m}, \psi^{m}\right) \\
& z^{m+1}=\underset{z}{\operatorname{argmin}} \quad \mathcal{L}_{\rho}\left(\gamma^{m+1}, z, \psi^{m}\right) \\
& \psi^{m+1}=\psi^{m}+\left(\gamma^{m+1}-z^{m+1}\right)
\end{aligned}
$$

In this formulation, each update/iteration, denoted by $m$, is separable and can be performed in parallel where:

\section{$\gamma$-update}

$$
\boldsymbol{\gamma}_{n}^{m+1}=\underset{\gamma}{\operatorname{argmin}} f_{n}\left(\boldsymbol{\gamma}_{n}\right)+(\rho / 2)\left\|\boldsymbol{\gamma}_{n}-\boldsymbol{z}_{n}^{m}+\boldsymbol{\psi}_{n}^{m}\right\|_{2}^{2}
$$

$z$-update:

$$
\begin{aligned}
\boldsymbol{z}_{n}^{m+1}=\underset{z}{\operatorname{argmin}} & \lambda \sum_{p}^{N_{s}} \sum_{k \in \Omega_{n j}}\left\|\gamma_{k}-z_{k}\right\|_{2} \\
& +(\rho / 2)\left\|\gamma_{n}^{m+1}-\boldsymbol{z}_{n}^{k}+\boldsymbol{\psi}_{n}^{m}\right\|_{2}^{2}
\end{aligned}
$$

$\psi$-update:

$$
\boldsymbol{\psi}_{n}^{m+1}=\boldsymbol{\psi}_{n}^{m}+\left(\boldsymbol{\gamma}_{n}^{m+1}-\boldsymbol{z}_{n}^{m+1}\right)
$$

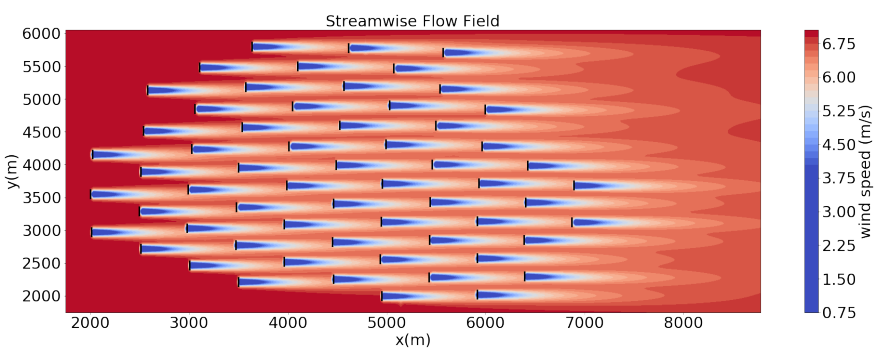

(a) Wind direction $=270^{\circ}$

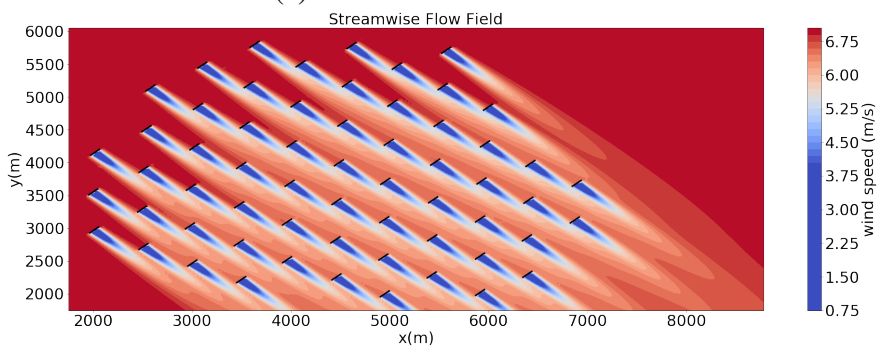

(b) Wind direction $=315^{\circ}$

Fig. 2: Steady-state flow field of the Princess Amalia wind farm computed using the wind turbine wake model described in Section II-A.

It is important to note that this is a nonconvex problem and each individual $\gamma$ and $z$ update is solved using standard gradient-based optimization algorithms. This paper uses the finite-difference gradient-based sequential least squares programming technique, commonly referred to as SLSQP [33].

\section{Simulation Setup}

To demonstrate the distributed optimization framework described above, we use the Princess Amalia wind farm [34]. This wind farm has 60 turbines that are simulated as the National Renewable Energy Laboratory's 5-MW turbine [35] encountering a wind speed of $U_{\infty}=7 \mathrm{~m} / \mathrm{s}$. We show results using two different wind directions to demonstrate the changing turbine interactions throughout the farm.

First, the wind direction is simulated from $270^{\circ}$ (west); see Fig. 2a. Here, the upstream turbines are heavily impacting downstream turbines. Subsets are easily defined in this case, with few interactions between subsets as shown in Fig. 3. The dark black lines indicate strong interactions between turbines. The lighter lines indicate weaker interactions. Only turbine 47 belongs to two subsets in this wind direction.

Second, the wind direction is simulated from $315^{\circ}$ (northwest), where the turbines have a smaller effect on downstream turbines, but each turbine affects more turbines. As a result, the subsets are larger and have more interconnections that must be taken into account when solving the distributed optimization problem.

\section{Optimization Results}

The distributed optimization algorithm was implemented on a desktop computer and run in serial. The distributed results presented in this section can be significantly sped up 


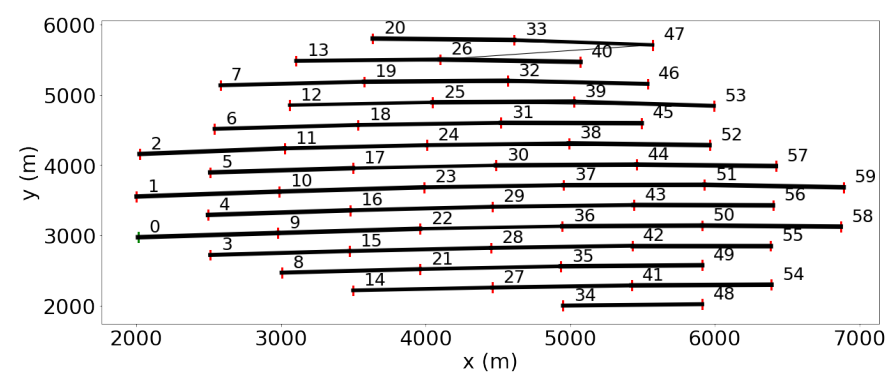

Fig. 3: Turbine interactions in a wind farm when the wind direction is $270^{\circ}$. The darker lines represent stronger interactions.

\begin{tabular}{|c||c|}
\hline Lead & Turbines in Subset \\
\hline \hline 0 & $9,22,36,50,58$ \\
\hline 1 & $10,23,37,51,59$ \\
\hline 2 & $11,24,38,52$ \\
\hline 3 & $15,28,42,55$ \\
\hline 4 & $16,29,43,56$ \\
\hline 5 & $17,30,44,57$ \\
\hline 6 & $18,31,45$ \\
\hline 7 & $19,32,46$ \\
\hline 8 & $21,35,49$ \\
\hline 12 & $25,39,53$ \\
\hline 13 & $26,40, \mathbf{4 7}$ \\
\hline 14 & $27,41,54$ \\
\hline 20 & $33, \mathbf{4 7}$ \\
\hline 34 & 48 \\
\hline
\end{tabular}

TABLE I: Subsets of turbines in the Princess Amalia wind farm when wind direction $=270^{\circ}$. Bold numbers indicate that that particular node is shared with another subset.

\begin{tabular}{|c||c|c|}
\hline Optimization & Performance Gain & Computation Time \\
\hline \hline Centralized & $17.8 \%$ & $4900 \mathrm{~s}$ \\
\hline Distributed & $17.7 \%$ & $102 \mathrm{~s}$ \\
\hline
\end{tabular}

TABLE II: Distributed algorithm performance with a wind direction of $270^{\circ}$.

by running the optimization in parallel. Future work will address this issue.

First, the Princess Amalia wind farm was optimized with a wind direction of $270^{\circ}$. Using Eq. (7), the weights between turbines, indicating the overall impact of each upstream turbine on a downstream turbine, were computed. The weights are shown in Fig. 3 for a wind direction of $270^{\circ}$ (top). Darker lines indicate that the interactions are strong. Lighter lines indicate that the interactions are weaker. Once the weights are determined, the wind farm is grouped into subsets. Table I shows the resulting subsets when the wind direction is $270^{\circ}$. The lead turbine indicates the turbine in the front of the subset relative to the wind direction. As shown in Table I, no subset contains more than six turbines (including the lead turbine). Optimizing each individual group is computationally inexpensive and significantly decreases the overall computation time. Table II shows the power gain computed using the distributed optimization algorithm compared with the centralized optimization problem described in Section IIB. The centralized system was solved using the same finite-

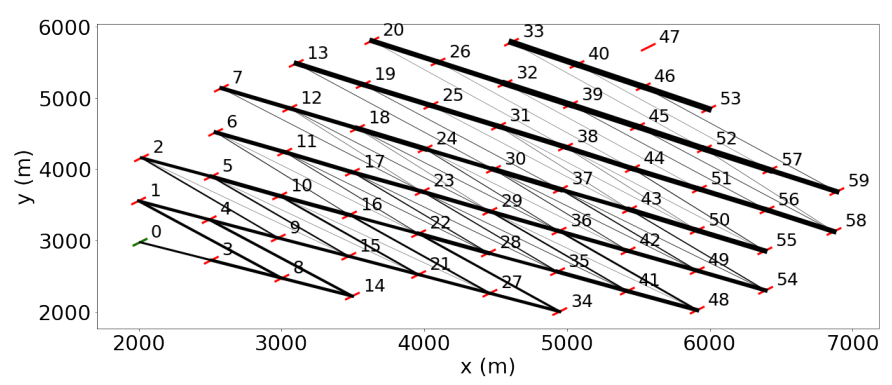

Fig. 4: Turbine interactions in a wind farm when the wind direction is $315^{\circ}$. The darker lines represent stronger interactions.

\begin{tabular}{|c||c|}
\hline Lead & Turbines in Subsets \\
\hline \hline 0 & $3, \mathbf{8}, \mathbf{1 4}$ \\
\hline 1 & $4, \mathbf{8}, \mathbf{9}, \mathbf{1 4}, \mathbf{1 5}, \mathbf{2 1}, \mathbf{2 7}, \mathbf{3 4}$ \\
\hline 2 & $5, \mathbf{9}, 10, \mathbf{1 5}, \mathbf{1 6}, \mathbf{2 1}, \mathbf{2 2}, \mathbf{2 7}, \mathbf{2 8}, \mathbf{3 4}, \mathbf{3 5}, \mathbf{4 1}, \mathbf{4 8}$ \\
\hline 6 & $11, \mathbf{1 6}, 17, \mathbf{2 2}, \mathbf{2 3}, \mathbf{2 7}, \mathbf{2 8}, \mathbf{2 9}, \mathbf{3 4}, \mathbf{3 5}, \mathbf{3 6}, \mathbf{4 1}, \mathbf{4 2}, \mathbf{4 8}, \mathbf{4 9}, \mathbf{5 4}$ \\
\hline 7 & $12,18, \mathbf{2 3}, 24, \mathbf{2 9}, \mathbf{3 0}, \mathbf{3 5}, \mathbf{3 6}, \mathbf{3 7}, \mathbf{4 1}, \mathbf{4 2}, \mathbf{4 3}, \mathbf{4 8}, \mathbf{4 9}, \mathbf{5 0}, \mathbf{5 4}, \mathbf{5 5}$ \\
\hline 13 & $19,25, \mathbf{3 0}, 31, \mathbf{3 7}, \mathbf{3 8}, \mathbf{4 2}, \mathbf{4 3}, \mathbf{4 4}, \mathbf{4 9}, \mathbf{5 0}, \mathbf{5 1}, \mathbf{5 4}, \mathbf{5 5}, \mathbf{5 6}, \mathbf{5 8}$ \\
\hline 20 & $26,32, \mathbf{3 8}, 39, \mathbf{4 4}, 45, \mathbf{5 0}, \mathbf{5 1}, \mathbf{5 2}, \mathbf{5 5}, \mathbf{5 6}, \mathbf{5 7}, \mathbf{5 8}, \mathbf{5 9}$ \\
\hline 33 & $40,46, \mathbf{5 2}, 53, \mathbf{5 7}, \mathbf{5 8}, \mathbf{5 9}$ \\
\hline 47 & None \\
\hline
\end{tabular}

TABLE III: Subsets of turbines in the Princess Amalia wind farm when the wind direction $=315^{\circ}$. Bold numbers indicate that that particular node is shared with another subset.

\begin{tabular}{|c||c|c|}
\hline Optimization & Performance Gain & Computation Time \\
\hline \hline Centralized & $0.78 \%$ & $2950 \mathrm{~s}$ \\
\hline Distributed & $0.52 \%$ & $257 \mathrm{~s}$ \\
\hline
\end{tabular}

TABLE IV: Distributed algorithm performance with a wind direction of $315^{\circ}$.

difference gradient-based optimization technique, SLSQP, as the distributed system. It can be seen that the distributed optimization produced similar results; however, the time to compute those results was 40 times less than in the centralized case. At a wind direction of $270^{\circ}$, there is significant potential for power gain - up to $17 \%$ by redirecting wakes using the yaw drive of the upstream turbines. It is important to note that the distributed results converge to the centralized results with a sufficient number of $m$ iterations of the ADMM algorithm described in Section III.

Next, simulations were run with a wind direction of $315^{\circ}$. Fig. 4 shows that there are numerous interactions between many different turbines. Further, the subsets defined in this case have many more overlapping turbines. The results of the subset groupings are shown in Table III. Again, the bold numbers indicate the overlapping turbines in each subset. The computational cost of the distributed algorithm is higher in the $315^{\circ}$ wind direction case because of the larger subsets and number of overlapping members of each subset. The larger number of iterations are required to ensure that the 
overlapping members converge to the same solution. The centralized system took less time than the previous case because there were weaker wake interactions that could not be controlled by wake redirection. In most cases, the wakes were sufficiently far to one side of the turbine. This relatively small amount of wake interaction is further demonstrated in the overall power gained in this scenario (i.e., less than 1\%). As with the previous case, the distributed algorithm results in similar performance gains as compared to the centralized system, again, at a fraction of the computational cost as indicated in Table IV.

\section{CONCLUSions}

This paper describes a computationally efficient distributed algorithm to maximize the power within a wind farm using wake redirection control. The results indicate that this algorithm can provide similar power gain results, but at a fraction of the computational cost when compared to solving a fully centralized optimization. Reducing the computational cost of the overall optimization allows for these wind farm control strategies to be deployed in real time. Future work will include moving toward dynamic modeling and closed loop control using this type of approach. In addition, we will be incorporating the trade-offs between increasing power and reducing loads in this framework to improve the overall performance of a wind farm.

\section{REFERENCES}

[1] L. Machielse, S. Barth, E. Bot, H. Hendriks, and G. Schepers, "Evaluation of heat and flux farm control-final report," ECN-E-07105, Energy Research Centre of the Netherlands (ECN), 2007.

[2] P. Gebraad, F. Teeuwisse, J. Wingerden, P. A. Fleming, S. Ruben, J. Marden, and L. Pao, "Wind plant power optimization through yaw control using a parametric model for wake effects - a CFD simulation study," Wind Energy, vol. 19, no. 1, pp. 95-114, 2016.

[3] P. A. Fleming, P. M. Gebraad, S. Lee, J.-W. van Wingerden, K. Johnson, M. Churchfield, J. Michalakes, P. Spalart, and P. Moriarty, "Evaluating techniques for redirecting turbine wakes using SOWFA," Renewable Energy, vol. 70, pp. 211-218, 2014.

[4] J. Meyers, W. Munters, and J. Goit, "A framework for optimization of turbulent wind-farm boundary layers and application to optimal control of wind-farm energy extraction," in 2016 American Control Conference (ACC). IEEE, 2016, pp. 519-524.

[5] R. J. Barthelmie, S. C. Pryor, S. T. Frandsen, K. S. Hansen, J. Schepers, K. Rados, W. Schlez, A. Neubert, L. Jensen, and S. Neckelmann, "Quantifying the impact of wind turbine wakes on power output at offshore wind farms," Journal of Atmospheric and Oceanic Technology, vol. 27, no. 8, pp. 1302-1317, 2010.

[6] K. E. Johnson and N. Thomas, "Wind farm control: addressing the aerodynamic interaction among wind turbines," in 2009 American Control Conference. IEEE, 2009, pp. 2104-2109.

[7] P. Fleming, J. Annoni, J. J. Shah, L. Wang, S. Ananthan, Z. Zhang, K. Hutchings, P. Wang, W. Chen, and L. Chen, "Field test of wake steering at an offshore wind farm," Wind Energy Science, vol. 2, no. 1, 2017.

[8] J. Quick, J. Annoni, R. King, K. Dykes, P. Fleming, and A. Ning, "Optimization under uncertainty for wake steering strategies: Preprint," National Renewable Energy Laboratory (NREL), Golden, CO United States, Tech. Rep., 2017.

[9] C. L. Bottasso, S. Cacciola, F. Campagnolo, and J. Schreiber, "Wake detection for wind farm control-formulation and validation," in 34th Wind Energy Symposium, 2016.

[10] F. Campagnolo, V. Petrović, C. L. Bottasso, and A. Croce, "Wind tunnel testing of wake control strategies," in 2016 American Control Conference (ACC). IEEE, 2016, pp. 513-518.
[11] S. Ferrari, G. Foderaro, P. Zhu, and T. A. Wettergren, "Distributed optimal control of multiscale dynamical systems: a tutorial," IEEE Control Systems Magazine, vol. 36, no. 2, pp. 102-116, 2016.

[12] K. H. Movric and F. L. Lewis, "Cooperative optimal control for multiagent systems on directed graph topologies," IEEE Transactions on Automatic Control, vol. 59, no. 3, pp. 769-774, 2014.

[13] J. Shamma, Cooperative control of distributed multi-agent systems. John Wiley \& Sons, 2008.

[14] Y. Zhang, M. Hong, E. Dall' Anese, S. Dhople, and Z. Xu, "Distributed controllers seeking AC optimal power flow solutions using ADMM," IEEE Transactions on Smart Grid, 2017.

[15] J. R. Marden, S. D. Ruben, and L. Y. Pao, "Surveying game theoretic approaches for wind farm optimization," in Proceedings of the AIAA aerospace sciences meeting. Nashvile, TN, USA, 2012, pp. 1-10.

[16] V. Spudić, C. Conte, M. Baotić, and M. Morari, "Cooperative distributed model predictive control for wind farms," Optimal Control Applications and Methods, vol. 36, no. 3, pp. 333-352, 2015.

[17] M. Vali, V. Petrovic, S. Boersma, J.-W. v. Wingerden, and M. Kuhn, "Adjoint-based model predictive control of wind farms: Beyond the quasi steady-state power maximization," Preprints of the International Federation of Automatic Control World Congress, Jul. 2017.

[18] M. Soleimanzadeh, R. Wisniewski, and K. Johnson, "A distributed optimization framework for wind farms," Journal of Wind Engineering and Industrial Aerodynamics, vol. 123, pp. 88-98, 2013.

[19] C. Bay, J. Annoni, T. Taylor, L. Pao, and K. Johnson, "Active power control for wind farms using distributed model predictive control and nearest neighbor communication," in American Control Conference (ACC), 2018. IEEE, 2017, p. Submitted.

[20] S. Boyd, N. Parikh, E. Chu, B. Peleato, and J. Eckstein, "Distributed optimization and statistical learning via the alternating direction method of multipliers," Foundations and Trends in Machine Learning, vol. 3, no. 1, pp. 1-122, 2011.

[21] P. Fleming, P. Gebraad, J.-W. van Wingerden, S. Lee, M. Churchfield, A. Scholbrock, J. Michalakes, K. Johnson, and P. Moriarty, "The SOWFA super-controller: A high-fidelity tool for evaluating wind plant control approaches," in Proceedings of the EWEA Annual Meeting, Vienna, Austria, 2013.

[22] M. Bastankhah and F. Porté-Agel, "A new analytical model for windturbine wakes," Renewable Energy, vol. 70, pp. 116-123, 2014.

[23] — "Experimental and theoretical study of wind turbine wakes in yawed conditions," Journal of Fluid Mechanics, vol. 806, pp. 506-541, 2016.

[24] A. Niayifar and F. Porté-Agel, "A new analytical model for wind farm power prediction," in Journal of Physics: Conference Series, vol. 625, no. 1. IOP Publishing, 2015, p. 012039.

[25] D. Dilip and F. Porté-Agel, "Wind turbine wake mitigation through blade pitch offset," Energies, vol. 10, no. 6, p. 757, 2017.

[26] M. Abkar and F. Porté-Agel, "Influence of atmospheric stability on wind-turbine wakes: A large-eddy simulation study," Physics of Fluids, vol. 27, no. 3, p. 035104, 2015.

[27] S. B. Pope, "Turbulent flows," 2001.

[28] T. Burton, D. Sharpe, N. Jenkins, and E. Bossanyi, Wind energy handbook. John Wiley \& Sons, 2001.

[29] L. Y. Pao and K. E. Johnson, "A tutorial on the dynamics and control of wind turbines and wind farms," in American Control Conference, 2009. IEEE, 2009, pp. 2076-2089.

[30] M. Newman, Networks: an introduction. Oxford university press, 2010.

[31] R. Xu and D. Wunsch, "Survey of clustering algorithms," IEEE Transactions on neural networks, vol. 16, no. 3, pp. 645-678, 2005.

[32] D. Hallac, J. Leskovec, and S. Boyd, "Network lasso: Clustering and optimization in large graphs," in Proceedings of the 21th ACM SIGKDD international conference on knowledge discovery and data mining. ACM, 2015, pp. 387-396.

[33] J. Nocedal and S. J. Wright, Sequential quadratic programming. Springer, 2006.

[34] P. A. Fleming, A. Ning, P. M. Gebraad, and K. Dykes, "Wind plant system engineering through optimization of layout and yaw control," Wind Energy, vol. 19, no. 2, pp. 329-344, 2016.

[35] J. Jonkman, S. Butterfield, W. Musial, and G. Scott, "Definition of a 5mw reference wind turbine for offshore system development," National Renewable Energy Laboratory, Golden, CO, Technical Report No. NREL/TP-500-38060, 2009. 